\title{
Rites de passage et récits de conversion : le témoignage de quelques anglaises au milieu du XVIle siècle
}

Claire Gheeraert-Graffeuille

\begin{abstract}
The breakdown of age-old rites of passage resulting from the collapse of the Church of England in the middle of the seventeenth century, led to the displacement and interiorization of ritual practices. The conversion narratives written by women from Congregationalist and Baptist churches show how rituals marking the stages of human life were spiritualised and transposed to inner life. When used as admission narratives, these testimonies became true rites of passage in the anthropological sense of the term, even overshadowing the traditional rite of baptism. The writing of conversion was used to ritualise and to commemorate this radical change, leaving traces or relics of conversion.
\end{abstract}

\section{Résumé}

La crise des rites de passage séculaires que provoque le démantèlement de l'Église d'Angleterre au milieu du XVIle siècle, conduit à l'intériorisation des pratiques rituelles. Les récits de conversion composés par des femmes issues des Églises congrégationalistes et baptistes, montrent comment des pratiques naguère inscrites dans les grands rythmes de l'existence sont spiritualisées et transposées à la vie intérieure. Lorsqu'ils servent de récits d'admission, ces témoignages tiennent lieu de rites de passage au sens anthropologique du terme, au point de se substituer au rite traditionnel du baptême. L'écriture autobiographique ritualise et commémore le passage en laissant sur le papier des traces ou des vestiges de la conversion.

\section{Citer ce document / Cite this document :}

Gheeraert-Graffeuille Claire. Rites de passage et récits de conversion : le témoignage de quelques anglaises au milieu du XVIle siècle . In: XVII-XVIII. Revue de la société d'études anglo-américaines des XVIle et XVIIle siècles. N62, 2006. Âges de la vie et rites de passage. pp. 19-34;

doi : https://doi.org/10.3406/xvii.2006.2408

https://www.persee.fr/doc/xvii_0291-3798_2006_num_62_1_2408

Fichier pdf généré le 21/12/2018

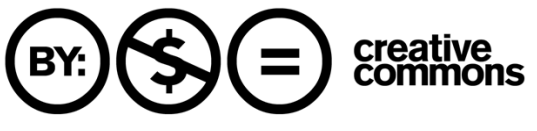




\section{RITES DE PASSAGE ET RÉCITS DE CONVERSION : LE TÉMOIGNAGE DE QUELQUES ANGLAISES AU MILIEU DU XVIIE SIÈCLE}

Résumé : La crise des rites de passage séculaires que provoque le démantèlement de l'Église d'Angleterre au milieu du XVIIe siècle, conduit à l'intériorisation des pratiques rituelles. Les récits de conversion composés par des femmes issues des Églises congrégationalistes et baptistes, montrent comment des pratiques naguère inscrites dans les grands rythmes de l'existence sont spiritualisées et transposées à la vie intérieure. Lorsqu'ils servent de récits d'admission, ces témoignages tiennent lieu de rites de passage au sens anthropologique du terme, au point de se substituer au rite traditionnel du baptême. L'écriture autobiographique ritualise et commémore le passage en laissant sur le papier des traces ou des vestiges de la conversion.

Abstract : The breakdown of age-old rites of passage resulting from the collapse of the Church of England in the middle of the seventeenth century, led to the displacement and interiorization of ritual practices. The conversion narratives written by women from Congregationalist and Baptist churches show how rituals marking the stages of human life were spiritualised and transposed to inner life. When used as admission narratives, these testimonies became true rites of passage in the anthropological sense of the term, even overshadowing the traditional rite of baptism. The writing of conversion was used to ritualise and to commemorate this radical change, leaving traces or relics of conversion.

Pour beaucoup d'hommes et de femmes au milieu du XVIIe siècle, la conversion est l'événement le plus important de la vie chrétienne. Aux yeux des calvinistes, ce passage de l'état de péché à celui de sainteté est une seconde naissance dans laquelle le croyant reçoit l'assurance du salut et le don divin d'une grâce efficace et 
gratuite (free grace). ${ }^{1}$ Le converti a le devoir de louer Dieu pour cette révélation, dans ses prières et ses méditations, bien sûr, mais aussi dans son journal ou encore dans ce que l'histoire littéraire a désigné au XIXe siècle sous le terme d' " autobiographie spirituelle » (Ebner 18-19) et dont l'exemple canonique, après les Confessions de saint Augustin, est le livre de John Bunyan, Grace Abounding to the Chief of Sinners (1666). ${ }^{2}$ Parmi les auteurs de récits de conversion publiés, on compte plusieurs femmes, issues pour la plupart des Églises baptistes et indépendantes qui fleurissent pendant la Révolution anglaise et qui subsistent clandestinement après la Restauration. ${ }^{3}$ Il s'agit en particulier de Jane Turner, d'Anna Trapnel, de Katherine Sutton et de Sarah Davy, quatre baptistes dont les autobiographies ont été publiées de leur vivant ou juste après leur mort. Des récits analogues, souvent beaucoup plus courts, sont aussi requis pour l'admission dans les Églises indépendantes ou baptistes, qu'on appelle aussi «sectes» selon la terminologie de Troeltsch. ${ }^{4}$ Il reste quelques traces de ces témoignages dans les florilèges constitués par des pasteurs dans années 1650, en particulier ceux de Samuel Petto, de Henry Walker et de John Rogers. Certes, ces récits de conversion féminins ressemblent beaucoup, sur le plan littéraire, aux écrits masculins composés à la même époque, mais le fait même qu'ils soient l'œuvre de femmes, et qu'ils manifestent ainsi l'égalité spirituelle prônée par protestantisme radical, justifie que l'on se

1. La définition calviniste de la conversion, adoptée par le Synode de Dort, s'applique à tous les récits de conversion étudiés ici : "And this is the regeneration, the new creation, the raising from the dead, and the making alive so clearly proclaimed in the Scriptures, which God works in us without our help [...] it is an entirely supernatural work, one that is at the same time most powerful and most pleasing, a marvelous, hidden, and inexpressible work, which is not lesser than or inferior in power to that of creation or of raising the dead». Voir article $12 \mathrm{du}$ Synode de Dort $<$ http://www.reformed.org/documents/canons_of_dordt.html $>$.

2. La publication des autobiographies s'accroît à partir de $16 \overline{50}$ (Watkins 26-28).

3. Le genre du récit de conversion se développe plus particulièrement chez les Particular Baptists, c'est-à-dire les baptistes calvinistes (par opposition aux General Baptists qui croient que la foi ouvre la voie à une rédemption universelle), chez les congrégationalistes ou indépendants, dont la foi est strictement calviniste ainsi que chez les presbytériens (dont aucun exemple ne figure ici).

4. «La secte est une communauté volontaire, dans laquelle on entre quand on veut. [...] l'individu n'est pas né dans la secte, mais il y entre à l'issue d'une conversion consciente » (Troeltsh cité par Cottret 235). 
concentre sur ce corpus singulier (Irwin xiii-xvii et GheeraertGraffeuille 32-34). ${ }^{5}$

L'objet de la présente enquête est de se demander dans quelle mesure il est possible d'appliquer à ces récits de conversion la définition que donne l'ethnologue Arnold Van Gennep du rite de passage, terme par lequel il désigne l'ensemble des pratiques préparant ou accompagnant le passage d'une personne d'un statut à un autre, d'un état à un autre (2-5). Le rapprochement est a priori aisé puisque le témoignage de conversion est l'élément le plus important de la cérémonie d'admission dans les Églises baptistes et indépendantes. Toutefois, l'assimilation de l'un à l'autre présente au moins deux difficultés. La première est que les protestantes radicales se méfient des dérives formalistes et que, par conséquent, la notion de « rite » entre en contradiction avec leur aspiration à une religion « déritualisée ». La seconde difficulté est que les récits de conversion sont rétrospectifs et que le changement qu'ils mettent en scène appartient au passé. Pour tenter d'éclaircir ces contradictions, à défaut de pouvoir les résoudre, on examinera d'abord le paradoxe selon lequel les récits de conversion, composés au sein de communautés hostiles à la liturgie traditionnelle de l'Église d'Angleterre, ritualisent la nouvelle naissance du croyant, vivifié par la grâce inconditionnelle du Christ. On verra ensuite pourquoi ces récits de conversion, lorsqu'ils servent de récit d'admission, répondent à la définition que donnent les anthropologues des rites de passage, et dans quelle mesure la cérémonie d'admission a alors tendance à se substituer au rite du baptême, désacralisé et souvent reporté. Enfin, on se demandera pourquoi l'écriture de la conversion, même lorsqu'elle n'a pas pour fonction d'assurer l'entrée dans une communauté, ritualise et commémore le passage.

Pendant la Révolution anglaise, les pasteurs disent de la conversion qu'elle est accomplie de façon ordinaire à travers les Écritures ou, de façon extraordinaire et directe, par des visions et des rêves. ${ }^{6}$ Dans tous les cas, l'Esprit-Saint est le véritable auteur de la

5. Le verset, cité par les défenseurs de l'égalité spirituelle, est Galates 3.28 : « There is neither Jew nor Greek, there is neither bond nor free, there is neither male nor female : for ye are all one in Christ Jesus ».

6. C'est une distinction que l'on retrouve dans les Églises indépendantes et 
conversion : «God [...] did preserve these experiences in her heart, where it seemeth they were written not with ink but by his holy spirit », lit-on dans la préface à l'autobiographie de Katherine Sutton (préface non paginée). Tous les auteurs de récits de conversion insistent sur la nature intérieure de l'expérience spirituelle. Selon le pasteur baptiste Vavasor Powell, "Experience [...] is the inward sense and feeling of what is outwardly read and heard » (172). Selon John Spilsbery, un autre pasteur baptiste, qui préface l'autobiographie de Jane Turner, la conversion doit permettre de découvrir « l'intérieur d'une âme qui a reçu la grace» (Turner, «To the Reader» [sig. $\mathrm{B} 3 \mathrm{v}])$. Le rejet du formalisme est un lieu commun de ces récits qui opposent sans cesse l'expérience intérieure à une religion " extérieure ». ${ }^{7}$ Pour ces auteurs, fortement marqués par le calvinisme, les rites, désignés par le terme biblique d' " ordinance », ont un statut similaire aux œuvres : leur observance est inutile avant que Dieu ne révèle directement sa grâce. La baptiste Katherine Sutton voit dans les religions établies des spectacles vides de Dieu: « outward performances is $[\mathrm{sic}]$ as the shell without the kernel» (25). Anna Trapnel ne cesse d'inviter ses coreligionnaires à recentrer leurs pratiques sur Dieu et non sur ses rites «Therefore, dear friends, let God be your all, and not Ordinances » (19). Mais ce rejet du rituel trouve malgré tout ses limites, car la convertie veut échapper à l'accusation d'antinomianisme ${ }^{8}$ qui menace ceux qui se méfient de l'institution et qui, à ce titre, sont suspectés de bafouer la loi morale. Sa principale stratégie de défense consiste à donner aux rites le rôle que les calvinistes accordent aux œuvres: les rites, comme les œuvres, sont un moyen de faire fructifier la grâce, et ainsi de sanctifier Dieu. Ils n'ont de sens qu'après la conversion (Nuttal, Holy Spirit 90-101). C'est pourquoi Anna Trapnel se défend de professer une doctrine qui servirait de caution au péché («a doctrine of liberty to $\sin » 13$ ) et énonce les règles d'un bon usage des rites (18-19); de même, Jane Turner affirme de façon très orthodoxe que les rites sont

baptistes aux tendances millénaristes. Voir Rogers 354 et Sutton sig. *2.

7. C'est une critique courante dans le protestantisme radical. La préface du Directory for Public Worship, de tendance presbytérienne, promulgué par l'Assemblée de Westminster, critique les cérémonies du Book of Common Prayer. Voir $<\mathrm{http}: / /$ www.covenanter.org/Westminster/directoryforpublicworship.htm $>$.

8. Doctrine selon laquelle la grâce salvifique dispenserait les élus d'observer la loi morale. 
les « fruits et les effets de la foi » et qu'ils constituent ainsi une forme de sanctification (76).

Dans la religion intérieure que préconisent les indépendants et les baptistes, il est aussi frappant de constater que beaucoup de rites sont transposés à la vie de l'âme : un examen de la structure et des images des récits de conversion montre, en effet, que la conversion est décrite comme une succession de rites de passage que l'âme doit surmonter. La tradition de lecture typologique de la Bible, à laquelle sont exercées ces protestantes, fournit de nombreuses images de passages, matériels ou géographiques, de sorte que l'histoire d'Israël devient une carte sur laquelle elles déchiffrent leur expérience. Pour Anna Trapnel, par exemple, la transition de la Loi à la grâce est décrite comme le passage du Mont Sinaï (lieu où fut conclue l'ancienne alliance), au Mont Sion (la ville sainte) (14), ou encore comparée à la traversée de la Mer Rouge (15). Jane Turner décrit le don de la grâce comme la sortie de Babylone et l'entrée dans Jérusalem (109). Pour Elizabeth Avery, dont le témoignage est recueilli par John Rogers, la conversion est une façon de revivre la sortie d'Égypte, et de passer ainsi de l'ancienne à la nouvelle alliance. ${ }^{9}$ À cette typologie, il faut ajouter des images scripturaires qui figurent les épreuves que doit subir l'âme en quête de son salut: le désert ("wilderness ») à traverser (Turner 117), les tempêtes à vaincre - ou pour reprendre les mots de Katherine Sutton, «through the valey of tears lieth the way to the mountain of joy » (11). Ces passages initiatiques sont pénibles, mais nécessaires, car ils préparent le chrétien à la réunion finale avec Dieu. Pour Anna Trapnel, le passage par la porte étroite est la récompense qui vient couronner de nombreuses tribulations : « this same power was a hand by divine appointment, leading me through varieties of inward bitter desolations, until it brought me not onely to the gates, but into a heaven of sweet consolation » (8). L'accès à la Jérusalem céleste est bien figuré comme le franchissement d'un seuil, assuré par un ange-portier : «O saints rejoyce with me; shall we be found worthy to enter in at the straight gate and plucked into the house by the angel of the New Covenant, when they without shall be stricken with blindness and not so able to fin the door?» (15). On retrouve ainsi, à travers ces images, les trois séquences dont parle

9. «But alas! I was yet under the Law, and Works, until God called me out of Egypt » (« Experience of Elizabeth Avery » 403). Voir aussi Turner 117. 
l'ethnologue Van Gennep à propos des rites de passage. La sortie d'un monde corrompu renvoie aux rites de séparation: comme l'enfant dont on coupe le cordon ombilical, par exemple, le pécheur qui se convertit se sépare de son milieu antérieur (Van Gennep 7273). Les épreuves qu'il doit ensuite subir évoquent les rites de marge, que l'on retrouve aussi bien dans les rituels d'adoption que dans ceux qui accompagnent l'admission dans une société secrète (114-15). Enfin, on peut considérer l'entrée glorieuse de l'âme dans la Jérusalem céleste - ou sa préfiguration - comme un exemple de rite d'agrégation, par exemple comme une version spirituelle des rites de la porte et du seuil, ou des rites de passage de frontières (19-33).

Cette ritualisation de la vie intérieure est encore assurée par la transposition métaphorique de rites de passage empruntés à la liturgie traditionnelle. Alors que l'efficacité du baptême est mise en cause pendant la Révolution par les baptistes et par les indépendants (Cressy 97-123), ${ }^{10}$ les femmes, issues de ces communautés, se servent paradoxalement de sa symbolique pour représenter la mutation que représente pour elles la conversion. Pour Anna Trapnel, la grâce qui apporte une vie nouvelle devient l'eau lustrale qui régénère le baptisé : «Free grace hath opened a Fountain to wash in, not only your feet, but heads and hands, yea your whole man ; in this fountain I was cleansed» (13). Étonnamment, la description qu'elle donne de l'illumination repose sur des images puisées dans la liturgie catholique. On trouve ainsi sous sa plume plusieurs références au vêtement blanc ${ }^{11}$ pour figurer la régénération ainsi qu'une allusion à l'onction du Saint-Chrême, qui fait des baptisés des rois, des prêtres et des prophètes aux yeux de Dieu :

Oh that Arras of glory, that now was my clothing ! now was I made like my Saviour, a crown given me, not made with pearls or rich diamonds, but far richer, not to be valued; earth cannot wear this crown, its onely the heaven of God that must injoy this prerogative, its those that are made Kings and Priests unto God, that are thus honoured. (11)

10. Dans les milieux indépendants et sectaires, le baptême est tout au plus un signe de la grâce. Sur la critique du baptême des enfants voir Sutton 7. Sur le baptême comme rite de passage, voir Van Gennep 89.

11. Voir Trapnel 15 («Saints clothing is the purest white»). Cette pratique repose sur la lecture d'Apocalypse 7.13, de Galates 3.27 et de Philippiens 3.21. 
De même, un autre rite de passage contesté pendant les années de la Révolution, le mariage, sert à représenter la conversion : les noces de l'âme avec le Christ sont la transition ultime vers la Jérusalem céleste. $^{12}$ C'est un motif récurrent dans l'autobiographie d'Anna Trapnel selon laquelle « the Saint that is thoroughly spiritual loves to walk inclosed in the arms of its Saviour» (16). Son autobiographie est ponctuée par des constats lyriques de cette union : "Christ is thine and thou art his » $(18,10)$, parfois modulés en « Christ was mine and I was his » (11). ${ }^{13}$ Pour elle, mais aussi pour la baptiste Anne Wentworth, les noces célestes importent davantage que le mariage terrestre ; le seul véritable époux, c'est le Christ : «Then was the full communion between Christ and my Soul, the Love knot, the comly bands of Marriage ; then did he espouse me unto himself for ever, and enable me to follow him, and give up my self as a thank-offering unto him » (12). L'union spirituelle - la conversion - vient remplacer ici un mariage malheureux avec une âme dure qui n'a pas fait l'expérience de la régénération intérieure. Le rejet du mariage terrestre au profit des noces célestes souligne à nouveau la primauté de la vie intérieure sur l'existence sociale; il suggère le désir d'échapper à l'autorité du mari, voire à celle du pasteur et du magistrat. Le rite de passage qui importe ici est spirituel : c'est l'union avec le Christ et non les noces humaines qui intéressent le converti. La présence obsédante de ces images conjugales dans les récits de conversion atteste ainsi du déplacement que subit la notion de rite de passage. Dans ces milieux calvinistes radicaux, l'accent est mis sur l'intériorisation et la spiritualisation de rituels naguère inscrits dans le cycle des saisons et dans les grands rythmes de l'existence.

Un autre paradoxe de ces récits, qui mettent en scène les grandes transitions du cheminement spirituel, est qu'ils sont intégrés à la procédure d'admission dans les Églises indépendantes et baptistes; ils constituent l'élément central de véritables cérémonies auxquelles les fidèles sont tenus de participer. ${ }^{14}$ Avant d'être membre à part

12. Le mariage devient un contrat civil obligatoire à partir du 29 septembre 1653 (Durston 68).

13. Voir aussi Davy, sig. [A4v] et Turner 2.

14. Patricia Caldwell en donne la définition suivante : « it was not a full spiritual autobiography but a separate, special and limited subgenre $»(78)$. 
entière de sa communauté de saints, le candidat doit, en effet, prouver à ses futurs coreligionnaires qu'il a reçu l'assurance du salut. Pour ce faire, il ne doit pas réciter un credo établi, comme c'est souvent le cas chez les presbytériens (Nuttall 112 et Caldwell 48-54); il lui faut décrire avec précision les œuvres de Dieu sur son âme, à la manière du Psalmiste : " come and hear all you that fear God, and I will tell you what he hath done for my soul » (Ps. 66.16). Ces témoignages sont prononcés devant toute la communauté ou, en cas de réticence, devant une assemblée plus restreinte (Nuttall 110 et Parr 10). La tripartition du rite de passage établie par Van Gennep permet encore d'éclairer le statut de ces témoignages : aux phases de séparation, de marge et d'agrégation correspondent le rejet du monde impur, la profession de foi et, enfin, l'entrée solennelle dans la fraternité (fellowship). L'intérêt de ce schéma herméneutique ${ }^{15}$ est qu'il permet de mieux comprendre la singularité des pratiques d'admission en vigueur dans les Églises congrégationalistes, non seulement en Angleterre, mais aussi dans le Nouveau Monde où, semble-t-il, elles étaient en usage depuis les années 1630 (Caldwell 54).

La profession de foi personnelle est l'élément principal de ce rite de passage ${ }^{16}$ Comme le résume le pasteur indépendant de Dublin, John Rogers, le converti doit fournir des "preuves» de sa foi : « Everyone to be admitted gives out some experimental Evidences of the work of grace upon his Soul (for the Church to judge of) whereby he (or she) is convinced that he is regenerate, and received of God » (354). Dans ces témoignages codifiés, le chrétien se sépare de sa vie antérieure : non seulement il se repent de ses péchés, mais il accepte de ne plus fréquenter les paroisses où se pressent des âmes impures. On reconnaît ici la première phase de séparation, dont parle Van Gennep dans son chapitre consacré aux rites d'initiation (137). Pour Katherine Sutton, par exemple, les premières appréhensions de la grâce lui font délaisser son ancienne Église : «Upon which I even melted in my spirit, and fell into a shedding of tears, resolving to separate from, and come no more to joyn in such a way of worship until I had very diligently searched into the true ways of God» (7).

15. Cette tripartition séquentielle a été jugée floue et trop universelle par certains historiens, mais elle n'en demeure pas moins opératoire ici. Voir Cressy 6.

16. Voir, par exemple, les mots de Sarah Davy : « it pleased God [...] to establish and confirm me more in the desires I had to joyn with the people of God in society, and enjoy Communion with them according to the order of the Gospel » (21). 
Cette séparation des purs et des impurs est conseillée par saint Paul qui demande aux chrétiens de Corinthe de ne pas se mêler aux incroyants (2 Cor. 6.17) : " wherefore come out from among them, and be ye separate, saith the Lord, and touch not the unclean thing " (Nuttall, Visible Saints 49-53). ${ }^{17}$ Ces versets figurent toujours en bonne place dans les covenants, qui servent de constitutions aux Églises, et que les nouveaux membres doivent aussi accepter le jour de leur admission. Pour les femmes, cette rupture, primordiale d'un point de vue spirituel, constitue aussi un acte d'indépendance sociale : l'appel de la conscience l'emporte sur l'obligation de se soumettre à ses supérieurs, qu'il s'agisse du magistrat, du père ou du mari (Parr 1). Pour les indépendants, la religion est en effet une affaire spirituelle, sur laquelle l'autorité des magistrats n'a en théorie pas de prise, et l'on voit ici pourquoi aux yeux des moralistes et des pasteurs des Églises établies, ces chrétiens aux tendances séparatistes - qui plus est quand il s'agit de femmes - sont suspectés de vouloir semer le désordre (Gheeraert-Graffeuille 56-57).

Dans ces expériences spirituelles, l'adieu au monde profane a toujours pour corollaire systématique le désir d'entrer en communion avec d'autres élus. On reconnaît ici la phase «liminale» ou «de marge » définie par Van Gennep. Lorsqu'elle rapporte les œuvres de Dieu sur son âme, la convertie est en effet dans une situation d'entre deux : elle rejette sa vie passée mais n'a pas encore été autorisée à se joindre à la fraternité (ou fellowship) des élus. C'est seulement lorsque l'assemblée de saints reconnaît l'authenticité de son expérience qu'elle peut s'agréger à sa nouvelle société et qu'elle change véritablement de statut. Dans l'expérience de Susanna Parr (Crawford 152-59), la cérémonie d'admission se confond avec le rituel de fondation de l'Église d'Exter (Parr 3). Chaque membre doit non seulement faire une profession de foi personnelle mais aussi souscrire à la constitution de l'Église en répondant à une liste de questions; autant d'éléments qui contribuent à faire du récit de conversion une démarche rituelle à part entière (Parr 3-4). Cependant, l'acceptation des nouveaux membres n'est pas systématique. Avant

17. Dans The Justification of the Independent Churches of Christ (London, 1641), Elizabeth Chidley, brillante polémiste, rappelle les fondements bibliques de la séparation: "God hath commanded all his people to separate themselves from all Idolatry [2 Cor. 6.14-17], and false worshipping [Rev. 14.9-12] and false worshippers [Rev. 18.4] ». Sur ce principe de séparation, voir Nuttall, Visible Saints 43-69. 
d'accueillir un nouveau croyant, la communauté doit se prononcer sur l'authenticité de son témoignage en vertu du « jugement de charité », c'est-à-dire avec la plus grande bienveillance possible $:^{18}$

we were every one of us both men and women to declare our thoughts of what was spoken; it being laid down as a ground, that we must have an account of a change from a naturall and legall estate, into an estate of grace and believing, of those whom we admitted into communion with us. I among the rest did according to my weak measure declare my selfe against that which I thought would not stand for grace. (Parr 10)

Cette obligation de juger la condition spirituelle de leurs « frères » et « sœurs» met les fidèles dans la délicate position d'apprécier les voies impénétrables du Très-haut. C'est un rôle que Susanna Parr compare à celui de «portiers » de la «Maison de Dieu ${ }^{19}$ et qui, selon les adversaires du congrégationalisme, n'est rien d'autre qu'une usurpation des prérogatives divines.

Ce qui distingue cette cérémonie d'admission de la liturgie traditionnelle, c'est qu'elle n'a pas l'efficacité magique souvent attribuée aux rites de passage. C'est un rituel probatoire dont le résultat demeure suspendu à l'avis des juges. C'est aussi un processus réversible qui peut aisément se retourner en son contraire, c'est-à-dire en rite d'excommunication, autre pratique en vigueur dans l'Église primitive, ${ }^{20}$ considéré par Van Gennep comme un rite de passage à l'envers, c'est-à-dire un rite d'exclusion (160): le chrétien qui a transgressé les règles de la communauté est renvoyé dans un monde impur, voire dans l'antichambre de l'enfer si l'on en croit Susanna

18. Voir en particulier Calvin, Institutions de la Religion chrétienne 4.1.8: "parce que le Seigneur voyait être expédient de savoir quels sont ceux que nous devons tenir pour ses enfants [...] Et d'autant qu'il n'était pas besoin en cela de certitude de foi, il a mis à la place un jugement de charité, selon lequel nous devons reconnaître pour membres de l'Eglise tous ceux qui par confession de foi, par bons exemples de vie et participation aux sacrements confessent un même Dieu et un même Christ avec nous ». Voir aussi Nuttall, Visible Saints 132 et Caldwell $46 \mathrm{ff}$.

19. «[W]e were to keep the house of God pure, we were set as Porters at the door, it was our duty [...], yea it was our liberty, that we, who were to have communion with those who came to be admitted, should give in our assent, or dissent in reference to their admission $\gg$ (Parr 11).

20. Dans la London Confession of Faith baptiste de 1647, l'excommunication est justifiée à partir de la Bible. La préservation de la pureté de ces Églises requérait un système disciplinaire rigoureux. Voir McGregor 45 et Acheson 57-58. 
Parr accusée, ainsi que sa compagne Mary Allein, d'avoir fréquenté une paroisse presbytérienne (Parr sig. [A4r] et Nuttall, Visible Saints 127). Aux yeux de ses condisciples, elle n'est que traître et rebelle (Parr 20), accusée d'avoir rompu le contrat qui la liait à la communauté et elle mérite par conséquent le sort réservé à la femme adultère (21). L'image d'une "Eglise tribunal» qui domine l'apologie de Susanna Parr ressort également du témoignage d'Anne Wentworth, bannie de sa communauté pour avoir quitté un mari qui la persécute $(8,16-17)$. Dans sa défense raisonnée, cette dernière considère que son excommunication résulte d'une perversion du « jugement de charité » (18); ses coreligionnaires, aveugles et impies, n'ont pas vu que le témoignage spirituel de son mari était une imposture : selon elle, il n'a jamais fait l'expérience d'une renaissance, ou, pour reprendre ses mots, " he never yet knew the new birth, the life of the new man» (7). Au lieu de rechercher les signes d'une grâce intérieure, les membres de la communauté se sont fiés aux apparences trompeuses de la conduite extérieure (1-2).

Une autre singularité de cette cérémonie d'admission est qu'elle a tendance à se substituer au baptême ou, du moins, à le précéder : d'une grande importance dans l'Église anglicane, ce sacrement ne disparaît pas dans ces communautés radicales, mais il perd la fonction sociale essentielle qu'il avait depuis le Moyen Âge ainsi que ses vertus régénératrices (Cressy 106-09 et Nuttall, Holy Spirit 96-98); au lieu de marquer l'entrée solennelle du jeune enfant dans l'Église et de le faire renaître dans la grâce, ${ }^{21}$ il n'est plus qu'une simple figure de l'alliance avec le Christ (Nuttall, Holy Spirit 118). Conformément à cette doctrine, les baptistes prédestianistes (Particular Baptists) administrent le baptême aux seuls convertis qui, pour la plupart, ont atteint l'âge adulte (McGregor 25-26 et 45). La baptiste Jane Turner relate comment son baptême ainsi que celui de son mari suivirent de près la cérémonie d'admission : «the next day we would go to the church [...] and declare what God had done in the great work of conversion, which accordingly we did, and gave them full satisfaction, and the week following were baptized and added to the

21. Sur la question de l'efficacité des rites, voir Cressy 110-11. Dans The Book of Common Prayer, la prière d'action de grâce prononcée au moment du baptême parle de « régénération ».

Voir $<$ http://justus.anglican.org/resources/bcp/1559/Baptism_1559. htm>. 
Church » (Turner 88 et Sutton 11). Ainsi, dans les Églises aux marges de l'orthodoxie calviniste, la logique de la foi prévaut sur le cycle naturel de la vie: le baptême, qui n'est pas indispensable au salut, passe au second plan. ${ }^{22}$ La vraie date de naissance du chrétien devient celle de sa conversion à la grâce inconditionnelle et gratuite du Christ - c'est un événement si important dans la vie du converti que celui doit sans cesse s'en souvenir et le commémorer.

L'écriture de la conversion n'est pas seulement motivée par le désir de devenir membre d'une secte. Elle constitue aussi un rituel qui figure en bonne place parmi les autres pratiques religieuses à travers lesquelles le chrétien doit sanctifier Dieu : l'écoute des sermons, la lecture de la Bible et sa méditation, la participation au culte, la prière. Le converti ne doit pas laisser son expérience spirituelle sous le boisseau, ou il encourt un châtiment divin ; c'est en tout cas ce que rapporte Katherine Sutton qui a négligé de publier son témoignage spirituel. Après avoir été punie dans son corps pour sa désobéissance (20), elle accepte finalement de répondre à l'appel de Dieu et de faire ressurgir le passé : «about three dayes after it was set upon my heart in the night, that I must writte my experiences [...] as soon as I set upon my work, it left mee, and I found the Lord [...] mightily assisting mee, in bringing things to my remembrance » (21).

Cette obligation à témoigner de son expérience est soulignée par les théologiens calvinistes comme William Perkins, Arthur Dent ou Robert Bolton qui dissèquent la psychologie de la conversion avec tant de soin que certains historiens n'hésitent pas à parler à propos de leurs anatomies de « morphologie de la conversion $» .{ }^{23}$ Les catégories théologiques qu'ils élaborent permettent en effet au croyant de décomposer l'expérience par nature fulgurante de sa conversion en phases logiques (Soubrenie 23-24). On trouve dans les autobiographies

22. Un texte important sur les tenants de la foi congrégationaliste, The Savoy Declaration (1658) rappelle : «Although it be a great sin to conterin or neglect this ordinance, yet grace and salvation are not so inseparably annexed unto it, as that no person can be regenerated or saved without it; or that all that are baptised are undoubtedly regenerated $»$.

23. Cette expression d'Edmund S. Morgan, Visible Saints: The History of a Puritan Idea (Ithaca: Cornell UP, 1963), est discutée par Patricia Caldwell (2, 39, 79). Ces étapes sont très présentes dans les récits de conversion. Voir, par exemple, Turner sig. B2v-B3 et Sutton 7. 
féminines du milieu du XVIIe siècle de nombreux échos à cette tradition (Ebner 31-49). Jane Turner évoque «the kind dealings of God before, in and after conversion" (titre) tandis que, dans une préface à ce témoignage, le pasteur John Spilsberry rappelle les différentes phases attendues du récit de conversion (sig. B2-B4). ${ }^{24}$ Anna Trapnel est aussi tout à fait consciente de cette grille de lecture puisqu'elle choisit de s'en démarquer afin de montrer que l'expérience de la conversion demeure irréductible à un cadre donné : « if I vary concerning some experience in this, in respect of doubting, and questioning union after sealing, it is my own experience, I must not record anothers experience » (12). À bien des égards, sa méfiance à l'égard d'une expérience préétablie recoupe la suspicion qui entoure les autres rites : à ses yeux, les conventions rhétoriques, comme les formes extérieures de dévotion, doivent céder le pas à une religion intérieure et spirituelle. En aucun cas, une "grille» théologique ne doit remplacer le témoignage personnel, ni la lettre tuer l'esprit. Il n'en reste pas moins que l'absence de méthode représente pour nos auteurs un écueil qu'il faut éviter à tout prix; un témoignage désordonné ne vaut pas mieux qu'une naissance prématurée, comme le souligne le pasteur Gardner : "Many persons are so drowned in confused and immethodical thought that all their intentions are like an untimely birth, or grass on the house top » (sig. [B5]).

Cette écriture rituelle de la conversion est en outre toujours rétrospective - elle commémore le passage, plutôt qu'elle ne l'accomplit. L'écriture est un travail de la mémoire auquel Dieu exhorte les hommes dans la Bible. ${ }^{25}$ Selon John Turner, La commémoration de ce passage du royaume des ténèbres vers celui du Christ, explique-t-il, est un devoir pour tout chrétien qui a reçu l'assurance du salut :

The right remembrance of our unconverted state will be a means to engage our hearts to be much in the thoughts of, and highly to prise, that unspeakable love and grace of our God through Jesus in quickning us who were dead in trespasses and sins, in translating us

24. Voir aussi la préface à l'autobiographie de Sarah Davy, sig [A6-A8].

25. C'est un des thèmes récurrents de la préface de Grace Abounding : " call to mind the former days, the years of ancient times; remember also your songs in the night » (Bunyan 3). Le passage cité est Nombres 33.1-2: «Moses [...] writ of the journeyings of the children of Israel, from Egypt to the land of Canaan; and commanded also, that they did remember their forty years' travel in the wilderness $»$. 
from the Kingdome of darkness into the Kingdome of his dear Son. (sig. [A3v]-A4)

Sans cette anamnèse, continue-t-il, tout le bénéfice de la conversion risque d'être perdu. Ne pas se souvenir de la grâce de Dieu, et donc ne pas persévérer dans la foi, c'est laisser la porte ouverte à Satan et à ses tentations (sig. [A4v]- [A5]).

Ainsi, l'écriture autobiographique ne réalise pas un passage celui-ci a déjà eu lieu - mais elle le ritualise et le commémore, en laissant des traces ou des vestiges de la conversion. Anna Trapnel parle de «legacy », c'est-à-dire de legs ou d'héritage (12), Sarah Davy de «pretious reliques » (page de titre); quant à Jane Turner, elle utilise le terme de "remembrancer» (43), et rappelle comment célébration et souvenir sont indissociables : " in the remembrance of [the former dealings of God towards me] I find my heart much engaged in praises to God» (46). À la façon d'un rituel de prière, l'écriture commémorative de la conversion resserre les liens entre le chrétien et Dieu.

Au milieu du XVIIe siècle, les récits autobiographiques féminins font ainsi apparaître que le démantèlement d'une religion nationale et la crise des rites de passage traditionnels ne conduisent pas à une disparition pure et simple des pratiques rituelles, mais plutôt à leur déplacement et à leur intériorisation. Non sans paradoxe, le récit de conversion, composé dans le cadre de communautés fermées, méfiantes à l'égard de tout formalisme, et où les rites ancestraux ne sont plus les temps forts de la vie paroissiale, métaphorise les étapes d'un cheminement de l'âme et réactive ainsi de l'intérieur des pratiques liturgiques qu'on aurait cru définitivement éliminées. Ainsi, dans les Églises indépendantes et baptistes, où ce n'est plus le baptême qui marque l'entrée du chrétien dans la communauté, le récit de conversion, prononcé lors d'une cérémonie, d'admission vaut comme rite de passage au sens plein du terme. Mais la fonction de ces témoignages dépasse la seule question de l'incorporation de nouveaux fidèles dans les groupes sectaires: au milieu du XVIIe siècle, ce sont tous les chrétiens, quel que soit leur sexe, qui sont encouragés à coucher sur le papier les progrès de la grâce dans leur âme; dans ce cadre plus large, l'écriture de la conversion, forme 
d'action de grâce tournée vers le passé, relève autant du rite de commémoration que du rite de passage.

Pour les femmes qui évoluent dans les communautés aux marges du protestantisme, la primauté de l'expérience intérieure, aussi bien dans les cérémonies d'admission que dans la foi quotidienne, représente un gain d'autonomie. Le rite n'est plus une pratique imposée de l'extérieur, mais le fruit d'une relation intime à Dieu, dans laquelle, au moins en théorie, les hommes n'ont pas le droit de s'immiscer. La dimension subversive et novatrice de cette religion, décrochée du rythme naturel de la vie et des saisons, se mesure à la virulence des nombreux pamphlets qui cherchent par tous les moyens à étouffer ces nouvelles voix féminines. En effet, à la différence des rites de passage traditionnels, en particulier ceux qui entourent la naissance et le mariage, les récits de conversion, œuvres du SaintEsprit, échappent au contrôle des autorités terrestres. C'est à ce titre qu'on peut les considérer comme un jalon important dans l'histoire de l'émancipation féminine.

\section{Claire GHEERAERT-GRAFFEUILLE \\ Université de Rouen}

\section{OUVRAGES CITÉS}

ACHESON, Robert J. Radical Puritans in England 1550-1660. London: Longman, 1990.

BunYan, John. Grace Abounding to the Chief of Sinners. Ed. W. R. Owens. London : Penguin, 1987.

CAldwell, Patricia. The Puritan Conversion Narrative : The Beginnings of American Expression. Cambridge : Cambridge UP, 1983.

CotTRET, Bernard. Histoire d'Angleterre XVI-XVIIIe siècles. Paris : PUF, 1996.

CRAWFORD, Patricia. Women and Religion in England. London : Routledge, 1993.

CRESSY, David. Birth, Marriage and Death : Ritual, Religion, and the LifeCycle in Tudor and Stuart England. Oxford : Oxford UP, 1997.

DAvY, Sarah. Heaven Realiz'd ; or, The Holy Pleasure of Daily Communion with God. London, 1670.

Durston, Christopher. The Family in the English Revolution. Oxford: Blackwell, 1989. 
EBNER, Dean. Autobiography in Seventeenth Century England: Theology and the Self. The Hague : Mouton, 1971.

GHEERAERT-GrafFEUILLE, Claire. La Cuisine et Forum : L'Émergence des femmes sur la scène publique pendant la Révolution anglaise (16401660). Paris : L'Harmattan, 2005.

IRWIN, Joyce L., ed. Womanhood in Radical Protestantism 1525-1675. New York : Edwin Mellen P, 1979.

MC GREGOR, J. F. « The Baptists : Fount of all Heresy ». Radical Religion in the English Revolution. Ed. J. F. McGregor and Barry Reay. Oxford: Oxford UP, 1984. 23-64.

NutTal, Geoffrey F. The Holy Spirit in Puritan Faith and Experience. Oxford : Blackwell, 1946.

- Visible Saints: The Congregational Way 1640-1660. Oxford: Blackwell, 1957.

PARR, Susanna. Susanna's Apology against the Elders. London, 1659.

PetTo, Samuel, ed. The Voice of the Spirit. London, 1654.

Rogers, John, ed. Ohel; or, Beth-shemesh. A Tabernacle for the Sun. London, 1653.

SOUBRENIE, Élisabeth. L'Art de la Conversion au XVIIe siècle : Étude sur les poètes métaphysiques anglais. Paris : Les Belles Lettres, 2004.

SutTon, Katherine. A Christian Woman's Experience of the Glorious Working of Gods Free Grace. London, 1663.

Trapnel, Anna. A Legacy for Saints; Being Several Experiences of the Dealings of God with Anna Trapnel. London, 1654.

TURNER, Jane. Choice Experiences of the Kind Dealings of God before, in and after Conversion. London, 1653.

Van GenneP, Arnold. Les Rites de passage. 1909. New York: Mouton, 1969.

WALKER, Henry, ed. Spiritual Experiences of Sundry Beleevers. London, 1653. Préface de Vavasor Powell.

WATKIns, Owen. The Puritan Experience: Studies in Spiritual Autobiography. London : Routledge and Kegan Paul, 1972.

Wentworth, Anne. A True Account of Anne Wentworths Being Cruelly, Unjustly and Unchristianly Dealt with by some of those People called Anabaptists. London, 1676. 